\title{
WIRELESS BILIARY STENT SYSTEM WITH WISHBONE-ARRAY RESONANT MAGNETOELASTIC (WARM) SENSOR AND CONFORMAL MAGNETIC LAYER
}

\author{
Scott R. Green ${ }^{l}$ and Yogesh B. Gianchandani ${ }^{1,2}$
}

${ }^{1}$ Department of Mechanical Engineering, University of Michigan, Ann Arbor, Michigan, USA

${ }^{2}$ Department of Electrical Engineering, University of Michigan, Ann Arbor, Michigan, USA

\begin{abstract}
Stents are used to maintain bile duct patency after duct narrowing occurs due to various pathologies. Unfortunately, stent placement results in sludge accumulation within a variable timeframe, leading to complications such as jaundice or liver damage. This paper presents a system for wirelessly monitoring the accumulation of sludge, comprising an integrated magnetoelastic system with a sensor and biasing permanent magnet layer that conform to the meshed topology and tubular curvature of a biliary stent. The sensors have an active area of 7.5 $\mathrm{mm} \times 29 \mathrm{~mm}$ and a mass of $9.1 \mathrm{mg}$. A $38 \%$ decrease in the resonant frequency - from $61.6 \mathrm{kHz}$ to $38.2 \mathrm{kHz}$ - after application of a sludge simulant totaling $20.9 \mathrm{mg}-2.3 \mathrm{X}$ the mass of the sensor - was measured with the integrated system.
\end{abstract}

\section{INTRODUCTION}

Stents are mesh tubular structures used to impart and maintain patency in a variety of vessels and ducts that have become constricted as a result of stenotic pathology. Though the act of implanting a stent relieves symptoms caused by the constriction, in-stent restenosis - a reappearance of the narrowing, typically due to the reaction of the body to the presence of the stent - is a risk associated with all stenting procedures.

An example of a stent application area - and the focus of this work - is the bile duct, which transports bile between the liver, gall bladder, pancreas, and small intestine. The constriction relieved by stent implantation is often due to pancreatitis, cholangitis, tumors, or gallstones. Restenosis can occur in an average of 4-5 months via formation of a bacterial matrix known as biliary "sludge" [1].

The timeframe for clinically significant restenosis to occur is highly variable from case to case. Current techniques for diagnosing a blockage are indirect and rely on detecting enzyme levels that may not increase until after the blockage is significant. The combined effect of the unknown pathogenesis time course and the indirect testing methods can result in either unnecessary, prescheduled interventions or in untimely interventions after patients exhibit outward symptoms of the blockage (and liver damage has already occurred). As such, a direct method of diagnosis - such as that shown in Fig. 1 - would enable timely intervention and eliminate unnecessary procedures.

We have previously reported on magnetoelastic wireless sensing of sludge accumulation utilizing externally applied AC interrogative and stent-integrated DC biasing magnetic fields [2]. The magnetic fields cause a magnetoelastic sensor integrated with the stent to resonate at a frequency that changes as local viscosity increases and as sludge accumulates. The mechanical resonance generates an oscillating magnetic field that can be measured with an external pick-up coil. Our previous work utilized discrete neodymium magnets to optimally bias the anisotropy of a ribbon sensor - i.e. a rectangular strip of magnetoelastic material - that was similar in design to sensors used in industrial/environmental applications [3-5]. Although this combination was shown to be effective in benchtop testing, the discrete nature of the magnet and sensor components leaves room for improvement - especially with regards to maintaining important distributed flexibility of the biliary stent. Components that conform to or mimic the open, flexible structure of the stent would lead to a system that is better

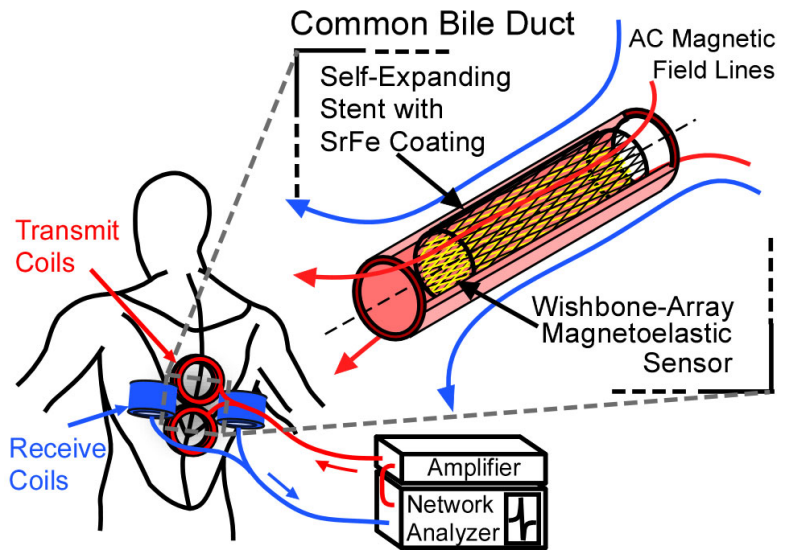

Figure 1: Conceptual diagram of in vivo magnetoelastic sensing of sludge accumulation for biliary stents.

able to withstand and accommodate the deformations required during catheter-based delivery, as well as lead to a system that preserves the structural functionality of the stent. With this viewpoint, this work focuses on an integrated magnetoelastic system with a sensor and biasing permanent magnet layer that conform to the meshed topology and tubular curvature of a biliary stent. Further, we investigate the impact of structural patterning, sensor shaping, and sensor material optimization.

\section{DESIGN AND MODELING \\ Wishbone-Array Sensor}

Biliary stents generally reach their final in situ diameter via an elastic self-expansion. This is in opposition to the plastic expansion of typical balloon-assisted cardiac stents. The need for large elastic diameter recovery in biliary stents leads to not only the utilization of materials with superior elastic properties (e.g. chrome-nickel Elgiloy or nickel-titanium Nitinol) but also to the use of open diamond-shaped patterns. Often these patterns are formed by braiding filaments into a tubular shape.

In keeping with the philosophy of mimicking the design of the stent with the design of the magnetoelastic sensor, we would like to use a material with superior elastic properties and to shape the material in diamond-shaped patterns. Fortunately, Metglas ${ }^{\mathrm{TM}}$ alloys are materials with excellent magnetostrictive properties as well as excellent elastic properties. For instance, the $2826 \mathrm{MB}$ alloy as used in this work is reported to have a yield strain of $1.6 \%$ [6], which is even higher than most cold-reduced Elgiloy yield strains of $\sim 1 \%$ [7]. Metglas ${ }^{\mathrm{TM}}$ is not, however, readily available in filament form. It is also likely that a resonant sensor fashioned from braided filaments would have low structural coupling and high damping at braid cross-over points, limiting efficiency as a resonator. For these reasons, both the stent and sensor in this work are batch-fabricated from foils of different base materials utilizing a photochemical machining (PCM) process [2]. As shown in Figure 2, an elongated wishbone-array pattern is used; this pattern allows good mechanical flexibility for both the stent and sensor while maintaining mechanical coupling and minimizing joint damping for efficient resonant operation of the sensor. To ensure that plastic strain in the sensor was avoided during deformation that is required during catheter-based delivery, an FEA model was 


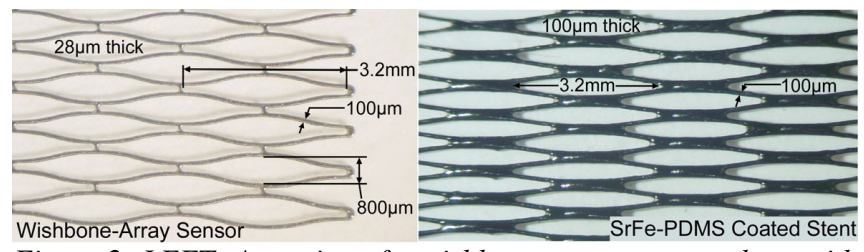

Figure 2: LEFT: A portion of a wishbone-array sensor, along with important dimensions. RIGHT: Stent with SrFe-PDMS coating.

utilized (Fig. 3). With the fabricated dimensions, FEA suggests that the wishbone-array sensor can undergo a 50\% reduction in circumference without plastic strain that may result in degradation of sensor performance.

Because the wishbone-array pattern represents a significant departure from typical ribbon sensors - which are analyzed for this application in detail in $[2,8]$ - we developed an FEA tool that is appropriate for estimating mode shapes and expected signal amplitudes from sensors with complicated structures. The crux of this tool is in its use of linearized constitutive equations describing the coupling between flux, field strength, stress, and strain in a magnetostrictive material:

$$
\begin{aligned}
& \vec{\sigma}=[C] \vec{\varepsilon}-\frac{[C][d]^{T}}{\mu_{o} \mu_{r}} \vec{B} \\
& \vec{H}=-\frac{[d][C]}{\mu_{o} \mu_{r}} \vec{\varepsilon}+\frac{1}{\mu_{0} \mu_{r}} \vec{B} .
\end{aligned}
$$

Equations (1) and (2) are versions of the so-called "piezomagnetic" equations - a name that highlights their similarity to piezoelectric equations - where $\sigma$ is the stress vector, $C$ is the stiffness matrix, $\varepsilon$ is the strain, $d$ is the magnetostrictivity matrix, $B$ is the magnetic flux density vector, $H$ is the field strength vector, $\mu_{0}$ is the permeability of free space, and $\mu_{r}$ is the relative permeability. Magnetostrictive materials are nonlinear, but linearization about an operating point in a resonant magnetoelastic analysis is prudent, with a rationale analogous to that used in small-signal models of transistor-based circuits. Equations (1) and (2) are implemented in this work utilizing COMSOL Multiphysics and coupled time-harmonic (frequency response) induction current and stress-strain modes. A detailed look at an FEA implementation for magnetostrictive materials is in [9]; the approach used in this work is modified for application to resonant sensors. In Figure 4, calculated mode shapes for planar wishbonearray sensors are shown. The mode shapes displayed are at frequencies corresponding to significant peaks in the measured frequency response for the planar sensors, with the mode shape at $61.6 \mathrm{kHz}$ resulting in the largest response amplitude. Note that the mode shapes combine significant longitudinal and transverse motion, whereas mode shapes of traditional ribbon sensors are

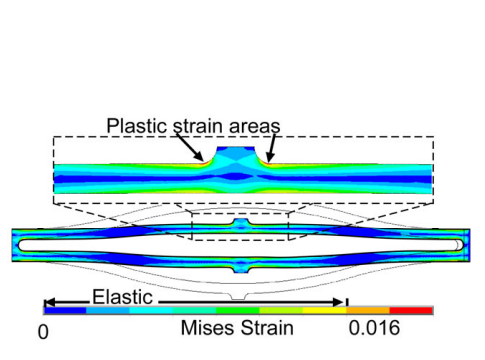

Figure 3: FEA calculated strain for a single wishbone cell. limited to longitudinal motion. The model also predicts signal amplitude trends for various sensor geometries.

\section{Conformal Magnetic Layer}

To achieve optimal magnetomechanical coupling, the magnetoelastic material must be biased with a DC magnetic field. This field offsets the as-fabricated anisotropy of the magnetic domains in the material, and the optimal field is dependent not only on the material of the sensor, but also on the feature sizes or aspect ratio of the final sensor. The process of selecting a bias field magnitude can be considered analogous to selecting an operating point for a transistor in an electrical circuit.

Our past work with integrated discrete magnets showed that sensor performance is improved when the bias field is as uniform as possible. This uniformity is difficult to achieve with integrated discrete magnets, because the field strength will necessarily decay as the distance from the magnets increases. However, if the magnetized portion of the system were to be continuously distributed, the field strength could be maintained more uniformly. This improves the sensor performance and eradicates high magnetic field gradients that lead to undesirable magnetic forces.

The distributed magnet is chosen in this work to be a layer of strontium ferrite $(\mathrm{SrFe})$ particles $(\sim 1 \mu \mathrm{m}$ average diameter, Hoosier Magnetics) suspended in polydimethylsiloxane (PDMS, Sylgard 184, Dow Corning). This choice is made again in keeping with minimally altering the functionality and structure of the biliary stent with the additional components. In this case, the polymersuspended particles can be applied in a thin, flexible layer conforming exactly to the stent structure (Fig. 2).

Other polymers have been used as a base for SrFe particles in microfabricated magnets described elsewhere [10]. SrFe particles have the advantages of being chemically inert (owing to their ceramic nature), and of being widely and inexpensively available in very small particle sizes. The chemical inertness is especially valuable in our implantable application. PDMS is chosen in this work due to its generally accepted biocompatibility and due to processing ease. In fact, the entire polymer-suspended magnet fabrication process (as described later) is preferable in terms of ease compared with alternative options such as sputtering or electrodeposition of a thin-film magnetic layer.

\section{FABRICATION \\ Wishbone-Array Sensor}

The wishbone-array sensors for this work are batch fabricated from a $28 \mu \mathrm{m}$ thick foil of $2826 \mathrm{MB}$ Metglas ${ }^{\mathrm{TM}}$ utilizing the PCM process. Feature sizes of the individual struts are $100 \mu \mathrm{m}$, which is near the feature size limit for the technology. The overall size of the active portion of the sensor (not including the anchor areas discussed later) is $7.5 \mathrm{~mm} \times 29 \mathrm{~mm}$, with a mass of $9.1 \mathrm{mg}$.

PCM is a planar process, so the as-fabricated sensors are also planar. Because the stent application calls for a tubular shape, and the lateral dimension of the sensor is larger than the diameter of the stent, the sensor must be curved into a tubular or semi-tubular shape to best match the stent geometry. Initial attempts to add curvature to the sensor via mechanical stress proved catastrophic for the sensor signal. Instead, the tubular shape is achieved in this work by placing the sensor against the inner wall of a fixture tube and annealing for 30 minutes. Various final radii can be achieved by either changing the fixture tube radius or by changing the anneal temperature. For instance, a $4.6 \mathrm{~mm}$ radius results from annealing at $375{ }^{\circ} \mathrm{C}$ for 30 minutes inside a $3.6 \mathrm{~mm}$ radius tube, while a $1.6 \mathrm{~mm}$ radius results from annealing inside a $1.25 \mathrm{~mm}$ radius tube. Lower temperatures lead to lesser final curvature. 


\section{Conformal Magnetic Layer}

To form the conformal magnetic layer, the PDMS is first mixed in a 10:1 base-to-curing-agent ratio. Subsequently, the $\mathrm{SrFe}$ particles are introduced in 1:1, 3:1, or 1:3 SrFe-to-PDMS by weight ratios and mixed in by hand until the mixture is consistent (usually about 1 minute of mixing time). The mixture is then poured or spread into a mold containing the stent. The stent is then pealed out of the mold, with a conformal layer of the magnetic suspension adhered due to surface tension. The layer is then cured for 30 minutes at $60{ }^{\circ} \mathrm{C}$. Thicker layers can be built up by repeating the process. Finally, the layer is magnetized uniformly along the long axis of the stent using a benchtop pulse magnetizer. In general, the 1:1 SrFe:PDMS ratio offered the best combination of workability and remnant strength of the ratios tested.

\section{Stent}

The stent is also batch fabricated using the PCM process, in this case from a $100 \mu \mathrm{m}$ thick foil of Elgiloy. As intended, the feature sizes and patterns are identical to those of the sensor (Fig. 2). The overall stent size is $5 \mathrm{~mm}$ (dia.) $\times 40 \mathrm{~mm}$.

\section{System Assembly}

Lateral portions of the wishbone-array sensor are connected to the active area with single struts. These areas act as anchors, and the single struts isolate the vibrating active area from the anchors. The anchors are bonded to the stent with a thin layer of PDMS.

Subsequently, the stent is rolled into a tubular shape and the resulting seam where the edges of the stent adjoin is also bonded with a thin layer of PDMS. The process is shown in Figure 5, and assemblies are shown in Figures 6 and 7.

\section{EXPERIMENTAL METHODS AND RESULTS Isolated Sensors}

Prior to integration, as-cast planar sensors were evaluated using a uniform but variable bias field applied by Helmholtz coils located coaxially with the long axis of the sensor. For all tests, a swept-frequency network analyzer signal was amplified and sent through a transmit coil, while the same analyzer measured the EMF generated on a receive coil. The sensors were located concentrically with these coils. Results from the initial evaluation for the largest modal response of four sensors are shown in Figure 8. The optimal bias field - where the amplitude of the response is largest $(10 \mathrm{mV})-$ is around $5 \mathrm{Oe}$. A clear dependence of resonant frequency on bias field can be seen - a manifestation of the $\Delta \mathrm{E}$ effect. The frequency and amplitude show repeatable performance across the tested sensors, as do frequencies and amplitudes from other modes, indicating a repeatable PCM fabrication process.

The sensors were then thermally treated either above $\left(375^{\circ} \mathrm{C}\right)$ or below $\left(325{ }^{\circ} \mathrm{C}\right)$ the material Curie temperature $\left(353{ }^{\circ} \mathrm{C}\right)$ and either remained planar or were given curvature. The resulting frequency characteristics are shown in Fig. 9. Post-treatment evaluation showed lower optimal biasing field $(\sim 1.5 \mathrm{Oe})$ and

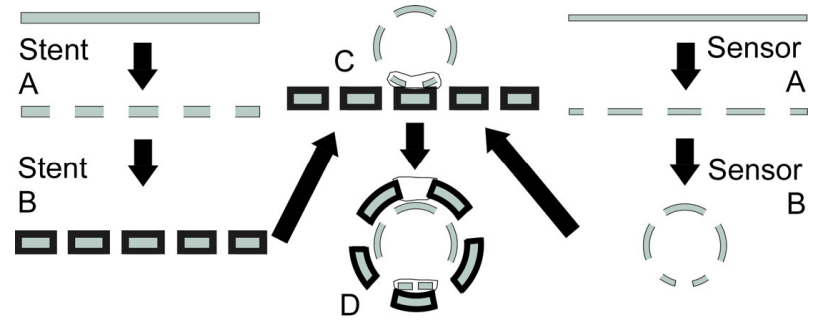

Fig. 5: Fabrication process. A) PCM patterning of Elgiloy (stent) and Metglas $^{T M}$ (sensor). B) Stent coated in SrFe-PDMS layer and magnetized. Sensor annealed in a tube. C) Sensor anchors bonded to stent with PDMS. D) Stent seam bonded with PDMS.
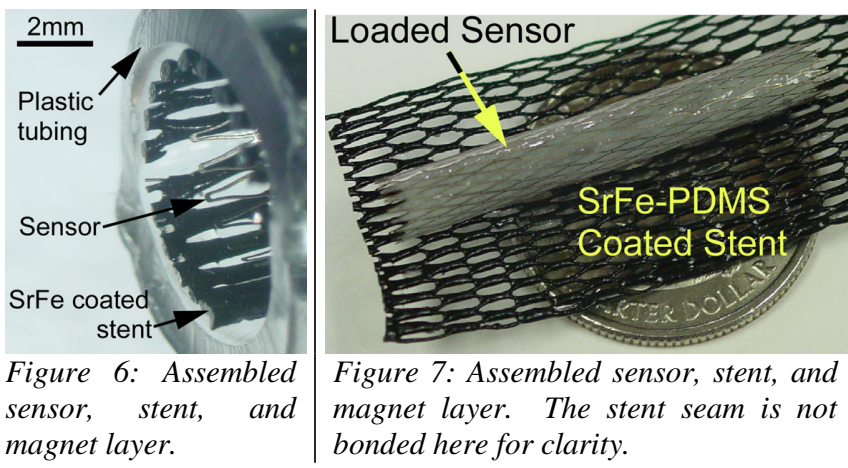

Figure 7: Assembled sensor, stent, and magnet layer. The stent seam is not bonded here for clarity.

improved signal level (up to $13.5 \mathrm{mV}$ p-p). This important result shows that thermal treatment facilitates thinner SrFe-PDMS layers, which simplifies fabrication and minimizes concerns about large chronically implanted magnetic fields.

As-cast and thermally treated sensors were compressed through $1.5 \mathrm{~mm}$ diameter tubes - a circumferential deformation of at least $37 \%$ - without signal degradation. The repeatable performance of this test across both as-cast and thermally treated sensors implies that the thermal treatment process does not lead to impaired mechanical properties. The slight discrepancy with the FEA model predictions may be due to an imperfect correlation between the onset of plastic strain and the onset of strains that change the magnetomechanical properties of the material.

Bile viscosity changes are precursors to sludge accumulation, so sensor response to viscosity was evaluated (Fig. 10). The tested viscosity range is much greater than the physiological range of bile (1-12 cP), but the results show that sensitivity and signal amplitude is maintained over a very large range that might be suitable for other applications. Note that a $2.5 \mathrm{~mm} \times 37.5 \mathrm{~mm}$ ribbon sensor resonant frequency will drop by only $6 \%$ over this viscosity range.

Accumulation of sludge results in a mass-loading effect on the sensor. This process was simulated by the application of two different materials - paraffin and a spray-on acrylate terpolymer to as-cast and thermally treated wishbone-array sensors, as well as to $2.5 \mathrm{~mm} \times 37.5 \mathrm{~mm}$ ribbon sensors. As shown in Figure 11, each of the sensor types reacts similarly in terms of resonant frequency to both sludge simulants. Further, the full scale range of each sensor type extends into the "critical zone", where accumulation begins to significantly narrow the cross-sectional flow area.

\section{Integrated System}

The integrated system, which consists of a curved wishbonearray sensor and a SrFe-PDMS coated stent, was evaluated in a manner similar to the isolated sensors but without the bias field supplied by the Helmholtz coils. In this way, all biasing of the sensor was provided by the conformal SrFe-PDMS magnetic layer. For the integrated system, sensitivity to viscosity over a physiologically appropriate range was measured even as mass was added. This experimental process showed that the normalized frequency response of the sensor to viscosity changes was not significantly affected by mass buildup (Fig. 12). Application of the acrylate terpolymer sludge simulant as a mass load showed that the frequency and signal amplitude of the integrated sensor reacted to mass loads similarly to those of the isolated sensors (Fig. 13).

\section{DISCUSSION}

Three important advantages of the wishbone-array sensor over typical ribbon sensors in this application are made clear by this work. First, the fine features sizes and large open area of the pattern present little obstruction to bile flow, which is the primary objective of a biliary stent. Second, the sensors are much more accommodating of the large deformations required for catheter- 


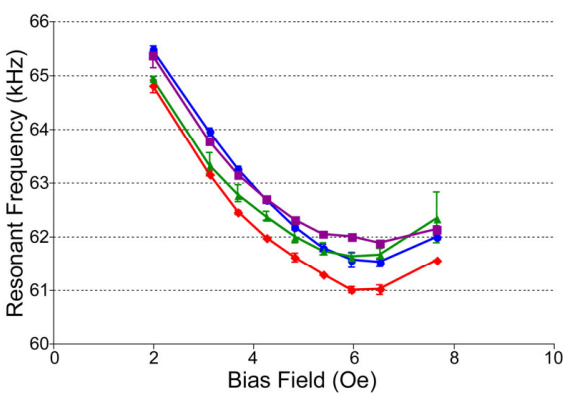

Figure 8: Evaluation of four as-cast planar wishbone-array sensors. The points in these figures represent the mean of three trials, and the error bars represent the minimum and maximum recorded values.

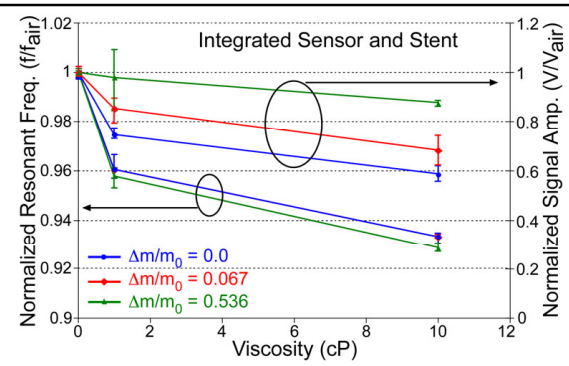

Figure 12: Response of the system to viscosity changes as mass accumulates.

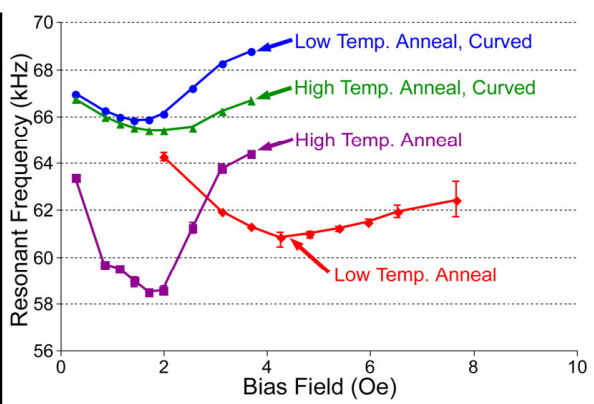

Figure 9: Characterization of wishbonearray sensors after thermal treatment.

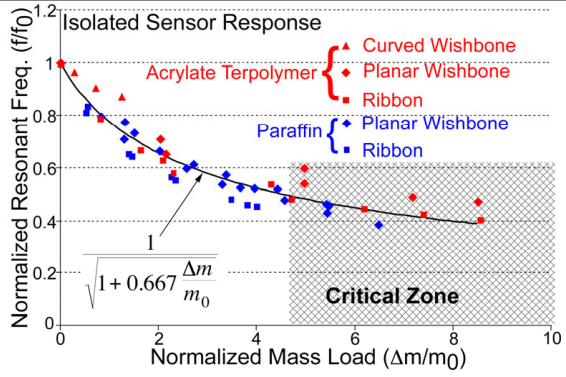

Fig. 11: Isolated wishbone and ribbon sensor response to mass loading from different sludge simulants. The curve is determined with a least-squares regression using the points and an equation of the form shown.

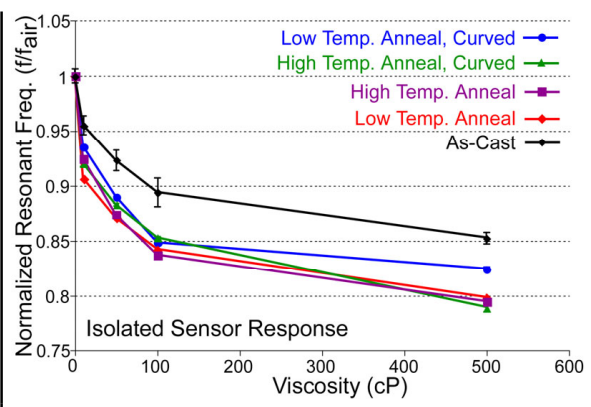

Figure 10: Isolated sensor response to changes in viscosity.

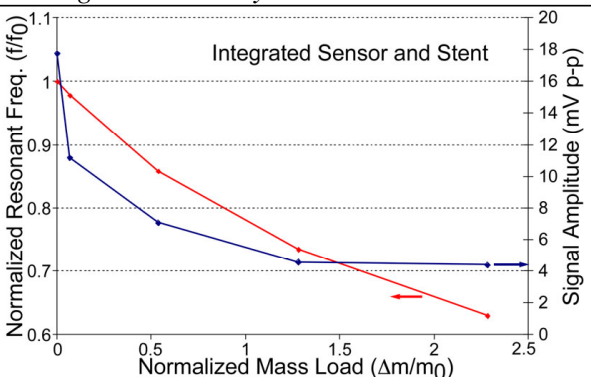

Figure 13: Response of the system to mass buildup. Mass added to the sensor was difficult to separate from mass added to the stent, so the equation for the curve in Fig. 11 was used to back-calculate the mass load. based delivery. Third, the sensors have a higher sensitivity to viscosity changes, which is a clinically relevant parameter in many pathological conditions [11]. The principal disadvantage of the wishbone-array sensor, at least with the present design, is the smaller signal amplitude. However, preliminary results show that the signal amplitude scales with the overall sensor length, so this disadvantage may be mitigated with a longer sensor design.

Prior to in vivo testing of the system, further evaluation of the mechanical properties of the stent must be done. Analytical models imply that the sludge simulants used in this work represent a worst-case scenario, as biofilms like sludge are likely to be less stiff than the test materials [12].

\section{CONCLUSION}

This work integrates a flexible wishbone-array magnetoelastic sensor and conformal magnetic layer with a biliary stent as a wireless system that monitors the stent environment. The system is sensitive to physiologically appropriate viscosity changes, showing a $7 \%$ decrease in resonant frequency in $10 \mathrm{cP}$ fluid. The system also is capable of measuring mass buildup that is associated with sludge accumulation, showing a $38 \%$ decrease in the resonant frequency after an applied mass load of $20.9 \mathrm{mg}$, or $2.3 \mathrm{X}$ the mass of the sensor. The integrated system is robust to deformations required for delivery and provides a uniform biasing layer that minimally affects stent mechanics. With appropriate scaling, the sensing methodology may be applicable in any stent, including cardiovascular and esophageal stents. Additionally, the improved viscosity sensitivity of the wishbone-array sensor may find use in industrial applications like monitoring oil refinement.

\section{ACKNOWLEDGEMENTS}

The authors acknowledge Dr. Grace Elta and Dr. Richard Kwon for discussions regarding stent usage. Mark Richardson assisted with test setup design and implementation. Metglas Inc., Hoosier Magnetics, and Dow Corning provided samples for this project. This work was supported in part by a NSF Graduate
Research Fellowship, the NSF ERC for Wireless Integrated Microsystems (WIMS), and the University of Michigan. Y. Gianchandani acknowledges support through the IR/D program while working at the National Science Foundation. The findings do not necessarily reflect the views of the NSF.

\section{REFERENCES}

[1] G. Donnelli, et al., "Plastic Biliary Stent Occlusion: Factors Involved and Possible Preventive Approaches," Clinical Medicine \& Research, Vol. 5, No. 1, 2007, pp. 53-60.

[2] S. R. Green, et al., "Photochemically Patterned Biliary Stents with Integrated Permanent Magnets and Deformable Assembly Features for Wireless Magnetoelastic Tissue Growth Sensing," Transducers 2007. June 2007, pp. 213-217.

[3] C. Grimes, et al., "Magnetoelastic Microsensors for Environmental Monitoring," IEEE Transducers 2001, pp. 278-281.

[4] M. Jain, et al., "A Wireless Micro-sensor for Simultaneous Measurement of $\mathrm{pH}$, Temperature, and Pressure," Smart Mat. and Struc., Vol. 10, 2001, pp. 347-353.

[5] C. Grimes, et al., "Simultaneous measurement of liquid density and viscosity using remote query magnetoelastic sensors", Rev. Sci. Inst., Vol. 7, Issue 10, 2000, pp. 3822-3824.

[6] J.-J. Lin, et al., "Embrittlement of Amorphous Fe40Ni38Mo4B18 Alloy by Electrolytic Hydrogen," Met. and Mat. Trans. A, Vol. 26, No. 1, 1995, pp. 197-201.

[7] www.matweb.com

[8] M.T. Richardson, et al., "Magnetoelastic Wireless Sensing of Tissue Growth for Self-Expanding Biliary Stents," IEEE MEMS 2007, pp. 469-472.

[9] J. Benatar, "FEM Implementations of Magnetostrictive-Based Applications", MS thesis, University of Maryland, 2005.

[10] L. Lagorce, et al, "Magnetic and Mechanical Properties of Micromachined Strontium Ferrite/Polyimide Composites," JMEMS, Vol. 6, No. 4, 1997, pp. 307-312.

[11] H. Zhang et al., "Role of Bile Mucin in Bacterial Adherence to Biliary Stents," J. Lab Clin Med, 139(1), pp. 28-34, Jan 2002.

[12] A.W. Cense, et al., "Mechanical Properties and Failure of Streptococcus mutans Biofilms, Studied Using a Microindentation Device," J. Microbiological Methods, Vol. 67, 2006, pp. 463-472. 